\title{
Éditorial: Les confessions d'une nouvelle rédactrice en chef
}

Assumer le poste de rédactrice en chef de la Revue canadienne du vieillissement constitue à la fois une expérience excitante et un exercice d'humilité. $\mathrm{Au}$ moment où j'écris cet éditorial, je suis sur le point de fêter mes trois premiers mois à ce poste. Il est de coutume de remercier les gens à la fin d'un mandat à un poste tel que celui-ci, mais je souhaiterais commencer par remercier un certain nombre de gens qui m'ont permis d'entamer ce mandat.

Je veux tout d'abord remercier Carolyn Rosenthal, la rédactrice en chef sortante, ainsi qu'Anne Marie Corrigan, notre directrice de rédaction aux Presses de l'Université de Toronto; leur collaboration et leur patience a permis de rendre la transition aussi aisée. Pour effectuer une telle transition, il ne suffit pas de transférer des dossiers d'un bureau à un autre. Je vous épargnerais les détails, mais disons qu'à plusieurs reprises Carolyn et Anne Marie m'ont éclairé de leur sagesse à un moment où j'en étais dépourvue. Je veux également remercier Carole Cohen, Daniel Lai, Thomas Hadjistavropoulos, Norm O'Rourke, Ingrid Connidis (chefs de rubrique) et Lucie Richard (rédactrice de comptes rendus) qui m'ont offert énormément de soutien grâce à leurs francs conseils au sujet des manuscrits que j'avais fait circuler et de certaines des idées que j'avais soulevées pour le journal. Enfin, je veux remercier les auteurs des manuscrits que j'ai étudiés jusqu'à présent. Je réapprends peu à peu l'importance de consulter des guides d'aide à la rédaction. Je suis consciente d'avoir demandé à certains auteurs d'apporter certains changements stylistiques qui n'étaient pas nécessaires et je suis également consciente que je continuerai probablement à formuler des demandes que les auteurs pourraient trouver contestables. Pour ce qui est du premier point, je vous présente mes excuses, et pour ce qui est du deuxième, n'hésitez pas à communiquer avec moi.

En ce début d'année 2005, la revue ne s'est jamais aussi bien portée. Comme l'a mentionné Carolyn dans son rapport présenté à l'assemblée générale annuelle tenue à Victoria, je souhaite rappeler aux lecteurs que la revue a finalement été intégrée, en 2004, à l'index Medline/Index Medicus. Il s'agissait d'un des objectifs à long terme de nombreuses personnes qui ont travaillé pour la revue. Nous leur sommes tous très redevables, mais Carolyn et Anne Marie, en particulier, méritent des félicitations pour cette réalisation. Pour citer un passage du rapport présenté par Carolyn, «le nombre total de soumissions est passé de 60 en 2002 à 68 en 2003 et laisse présager une augmentation encore plus subséquente cette année. En date du 24 octobre 2003, 53 manuscrits avaient été soumis. En date du 6 octobre 2004, 62 manuscrits avaient été soumis. Pour 2004, le total pourrait atteindre 75 à 80 manuscrits». L'objectif de Carolyn était d'atteindre une centaine de soumissions de manuscrits par année, et j'espère que nous atteindrons cet objectif en 2005. Cela signifie que chaque numéro de la revue comprendrait de huit à dix articles.

L'Institut du vieillissement des Instituts de recherche en santé du Canada ainsi que le Conseil de recherches en sciences humaines du Canada (CRSH) continuent de nous fournir un soutien financier, ce que nous apprécions beaucoup. À l'aube de l'année 2005, nous savons maintenant que nous disposons d'une nouvelle subvention à la publication de trois ans fournie par le CRSH et nous espérons obtenir un soutien constant de la part de l'Institut du vieillissement.

En tant que géographe, spécialiste des sciences sociales et gérontologue, j'ai beaucoup appris en lisant la Revue canadienne du vieillissement. J'en apprends encore plus maintenant, en tant que rédactrice en chef de votre revue. Dans tous les périodiques auxquels j'ai participé à titre de rédactrice, j'ai toujours été convaincue que les revues appartiennent aux auteurs et aux lecteurs, et que le rédacteur ou la rédactrice est là pour travailler pour eux, et pas l'inverse. Dans mes prochains éditoriaux, je souhaite entamer un dialogue sur la manière d'améliorer la revue au cours de mon mandat de rédactrice en chef. Je m'intéresse toujours aux opinions des auteurs et des lecteurs ainsi qu'à leurs conseils sur la manière d'accomplir un meilleur travail. N'hésitez pas à communiquer avec moi en tout temps, à l'adresse suivante: rosenber@post. queensu.ca. 